\title{
Asset Price Dynamics with Local Interactions under Heterogeneous Beliefs*
}

\author{
Valentyn Panchenko** \\ Department of Economics, Australian School of Business, University of New South Wales \\ Sydney, NSW 2052, Australia \\ Sergiy Gerasymchuk \\ Advanced School of Economics, University of Venice \\ Cannaregio 873, 30121 Venice, Italy \\ Oleg V. Pavlov \\ Department of Social Science and Policy Studies, Worcester Polytechnic Institute \\ 100 Institute Road, MA 01609-2280 Worcester, USA
}

February 24, 2010

\begin{abstract}
This paper investigates the effect of network structure on the asset price dynamics. We propose a simple present value discounted asset pricing model with heterogeneous agents. Every period the agents choose a predictor of the future price on the basis of past performance of their own and alternative strategies and form their demands for a risky asset. The information about the performance of an alter-

\footnotetext{
*We thank the participants of the workshop "Ten years of CeNDEF" for their comments and suggestions. We are also grateful to the organizers of the Seventh Trento Summer School in Agent-Based Computational Economics during which we began to work on this paper, for creating stimulating environment and for the opportunity to present our work. We thank Mikhail Anufriev, William Brock, John Duffy, Cars Hommes, Alan Kirman and Marco LiCalzi for their encouragements and suggestions. Authors are responsible for possible errors and omissions.

${ }^{* *}$ Corresponding author. Telephone: +61 2 93853363; E-mail: v.panchenko@unsw.edu.au.
} 
native strategy is available only locally from the directly connected agents. Using the rewiring procedure we produce four types of commonly considered networks: a fully connected network, a regular lattice, a small world, and a random network. The results show that the network structure influences asset price dynamics in terms of the region of stability and volatility. This is mostly due to the different speed of information transmission in the different networks.

Keywords: asset pricing, local interactions, networks, small world, heterogeneous beliefs, price dynamics.

JEL classification: C45, C62, C63, D84, G12. 


\section{Introduction}

To a large extent innovative ideas and practices diffuse in communities through interpersonal communication. Popular ideas in financial markets also often spread through conversations (Shiller, 1995). In a survey of institutional investors in the USA, Shiller and Pound (1989) found that money managers who invested in stocks with extremely high growth of the price/earnings ratio were often discussing their trades with colleagues. Arnswald (2001) found that among fund managers in Germany information exchange with other financial and industry experts was the second most important factor influencing their investment decisions, which complemented conversations with their colleagues and reports from media. Similarly, a study of fund managers by Hong et al. (2005) provided the strong support for the importance of informal communication. Household investment decisions are also affected by interpersonal communication. Madrian and Shea (2000) and Duflo and Saez (2002) showed that employees are more likely to join an investment retirement scheme if their colleagues have done so. By reviewing data from the Health and Retirement Study, Hong et al. (2004) suggested that interaction with neighbors or church attendance increased the likelihood of a household investing in stocks.

We study the impact that local interactions between investors have on the asset price dynamics. The innovation of our approach is to bring together ideas from three streams of literature: the rapidly developing literature on networks, the literature on heterogeneous agent models, and the literature on agent-based models. We explore a range of local interaction patterns by introducing different types of network topologies into the stylized heterogeneous agent model of Brock and Hommes (1998).

We find that not only communication influences asset price dynamics, but also the patterns of communication are important because they determine the speed of information exchange. The analysis of the statistical properties of the time series 
shows that the model with a small world communication structure generates the most realistic asset price dynamics among all other structures studied in this paper.

In the next section we survey heterogeneous beliefs models. The following section examines different network structures and their properties. Section 4 contains the description of the model and its application to the model of Brock and Hommes (1998). In Section 5 we analyze the model, present and discuss the results of the simulations. Section 6 concludes the paper.

\section{Bounded rationality and heterogeneity in asset pricing}

The rational expectations theory of finance (Friedman, 1953) asserts that rational investors would drive irrational traders out of the financial markets. Numerous empirical studies, however, showed that successful traders follow a variety of investment strategies (e.g. Frankel and Froot, 1987; Ito, 1990). DeLong et al. (1990) were among the first who analytically demonstrated that irrational noise traders may survive in the market with fully rational traders. The survival is possible because noise traders bear higher risk which leads to higher returns in the long run. Moreover, heterogeneity of expectations can explain asset prices dynamics. Day and Huang (1990), Chiarella (1992), Kirman (1993) and Lux (1995) showed that trades between different agents that follow simple behavioral rules lead to endogenous price fluctuations. Recently Alfarano and Milaković (2009) enriched the Kirman-Lux model with explicit network structures.

Brock and Hommes (1998) and Hommes (2001) introduced a structural asset pricing heterogeneous agent model (denoted the BH model henceforth) with an evolutionary switching between several trading strategies. The strategies differed only in the expectation about the future price of the risky asset. The fitness measure of each strategy was freely available to all the agents. The BH model showed that 
the rational expectations strategies do not necessarily drive out boundedly rational strategies. In fact, both types can co-exist in a market. Despite its complexity, the BH model had a closed-form analytical solution. The model was able to replicate excess volatility, a stylized fact which was not reproduced by the rational expectations models.

To improve the realism of the BH model, Anufriev and Bottazzi (2004) introduced heterogeneous investment horizons, while Anufriev and Panchenko (2006) investigated the changes in the model outcomes due to different market architectures. De Fontnouvelle (2000) enriched the model with various information flow schemes about the dividend payments. Brock et al. (2005) extended the BH model to many trader types, and Hommes (2002) modified the original BH model to reproduce volatility clustering. Hommes et al. (2005) included a market maker into the market pricing mechanism, while Brock et al. (2006) studied how the presence of risk hedging instruments in the form of Arrow securities affects the market dynamics. Boswijk et al. (2007) estimated the parameters of the BH model using annual US stock price data. Chang (2007) enriched the model with exogenous social interactions of the Brock and Durlauf (2001) type. Diks and van der Weide (2005) studied continuous distribution of the agent beliefs, while Gerasymchuk (2008) introduced prospect theory-like preferences of the agents into a modified BH model. For a detailed survey of the state of the art in the heterogeneous agent modeling see Hommes (2006).

Another approach used in financial market modeling relies on agent-based models. Agent-based models aim to re-create real stock markets with large populations of interacting artificial agents. Santa Fe artificial stock market (Arthur et al., 1997; LeBaron et al., 1999; Ehrentreich, 2006) is an example of such an approach. Other examples of agent-based models include Gode and Sunder (1997), Chen and Yeh (2001), Chen et al. (2001), and Duffy (2001). A major advantage of these models 
over the heterogeneous agent models is that they allow for higher flexibility, richer behavioral assumptions, and more realistic market architectures. This comes at the price of increased complexity. Closed form analytic solutions are typically not available for this class of models and computer simulations are used to analyze them. For further details on agent-based modeling in finance we refer an interested reader to the review by LeBaron (2006).

In this paper we combine the rapidly developing literature on networks with the literature on heterogeneous agent models by introducing local interactions into the stylized BH model. Due to high complexity of the resulting system, we lose analytical tractability and have to use agent-based-type simulations to investigate the model. Our aim is to study the effects of different types of local interactions on the asset price dynamics. In our setting the information about the fitness measure of each particular strategy is available to the agents only locally through other agents directly connected to them. We explore a range of local interactions patterns by introducing different types of networks. The analytically tractable $\mathrm{BH}$ model is a benchmark to which we compare our results.

\section{Social networks}

The analysis by Wasserman and Faust (1994) and Valente and Davis (1999) suggested that a typical social network has the following features (summarized in Watts, 1999): 1) there are many participants in the network; 2) each participant is connected to a small fraction of the entire network; in other words, the network is sparse; 3) even the most connected node is still connected only to a small fraction of the entire network; that is, the network is decentralized; 4) neighborhoods overlap; i.e. the network is clustered.

To capture these characteristics Watts and Strogatz (1998) introduced a network 


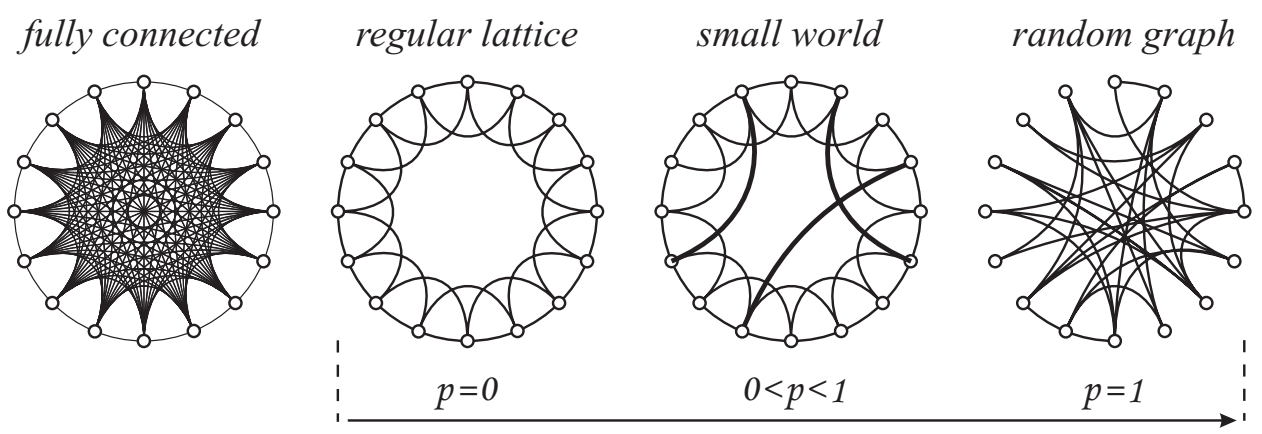

Figure 1: Network topologies (adapted from Watts and Strogatz (1998)).

model called a small world. It is an intermediate network between a regular lattice network, where the agents (called nodes in the network literature) are connected in a geometrically regular way, and a random graph, where the connections are random. Social scientists have recognized that the small world network is a good model approximating social interactions in real life. Networks with the small world properties include social networks of the US corporate elite (Davis et al., 2003), partnerships of investment banks in Canada (Baum et al., 2003), and many more. Small world networks emerge when participating agents form networks through a mix of random and strategic interactions (Baum et al., 2003 and Morone and Taylor, 2004).

Figure 1 shows four examples of network topologies. In the fully connected network, all nodes are linked to all other nodes. In the regular lattice, each node is connected to two nodes on each side, that is, each node has 4 connections, or edges. In order to form a small world network an edge is reconnected to a different randomly chosen node on the lattice (avoiding self- and double-connection) with a given rewiring probability, $0<p<1$. Such rewiring of the nodes continues until all the edges are processed. In the limit when $p=1$ the network becomes a random graph.

Watts and Strogatz (1998) suggested to characterize the structural properties of 


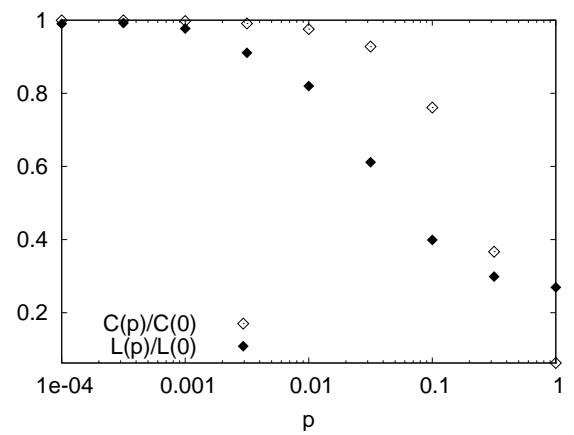

(a) $N=100$

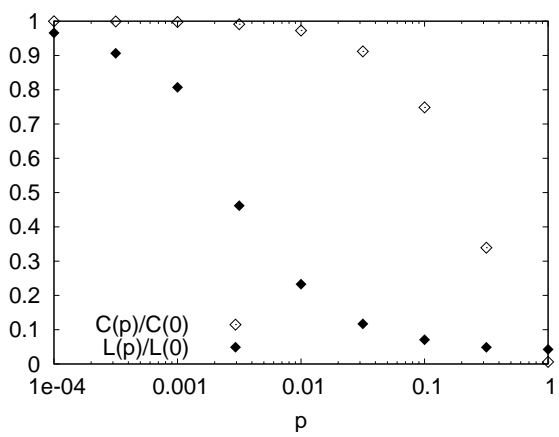

(b) $N=1000$

Figure 2: Clustering coefficient and characteristic path length for networks of different size. Note: logarithmic scale is used for abscissa.

the networks with two measures: a clustering coefficient, $C(p)$, and a characteristic path length, $L(p)$. The clustering coefficient of a node is calculated by dividing the number of edges between the node and its neighboring nodes by the maximum possible number of edges between them. It indicates how well the neighborhood of the node is connected or, in other words, it expresses the cliquishness of the neighborhood. By averaging over the clustering coefficients of all the nodes in a network we obtain the clustering coefficient of the network $C(p)$. The characteristic path length $L(p)$ measures the average separation between two nodes and is defined as the average number of edges in the shortest path between two nodes. This measure is inversely related to the average speed of local signal transmission between two nodes of the network. The shorter characteristic path length is, the faster the information spreads through the network.

For each value of the rewiring probability $p$ we obtain a network with new structural properties. These properties also depend on the number of nodes $N$. A small world network can be defined in terms of the clustering coefficient and characteristic path length. Specifically, it is a decentralized sparsely connected network with a high clustering coefficient $C(p)$ and a small characteristic path length $L(p)$. The values of the normalized clustering coefficients and the characteristic path lengths for 
different rewiring probabilities $p$ and two network sizes with $N=100$ and $N=1000$ nodes are depicted in Figure 2. Normalization is implemented over the corresponding characteristics of the regular lattice (for which $p=0$ ). The small world network properties are observed for $p=0.1$ when $N=100$ and for 0.01 when $N=1000$. This is consistent with Albert and Barabási (2002) who suggest that the rewiring probability leading to a small world network is inversely proportional to the number of nodes.

\section{Heterogeneous belief model with local interactions}

First, we briefly describe the BH model which we modify to allow for local interactions. ${ }^{1}$ There are two assets that are traded in discrete time: a risk-free asset paying a constant gross return $R_{f}=1+r_{f}$ and a risky asset paying a stochastic dividend $y_{t}$ at the beginning of each trading period $t$. The dividend is assumed to be independently and identically normally distributed (i.i.d.) with mean $\bar{y}$ and variance $\operatorname{Var}[y]$. The price $p_{t}$ per-share (ex-dividend) of the risky asset in period $t$ is obtained from the market clearing condition using a Walrasian auctioneer protocol. ${ }^{2}$ The wealth dynamics is:

$$
W_{t+1}=R_{f}\left(W_{t}-p_{t} z_{t}\right)+\left(p_{t+1}+y_{t+1}\right) z_{t}=R_{f} W_{t}+\left(p_{t+1}+y_{t+1}-R_{f} p_{t}\right) z_{t}
$$

where $W_{t}$ and $W_{t+1}$ are the wealth levels in period $t$ and $t+1$ correspondingly, and $z_{t}$ is the number of shares of the risky asset purchased at date $t$.

\footnotetext{
${ }^{1}$ While the $\mathrm{BH}$ model is presented in terms of deviations from the fundamental price, we present the model in terms of the price itself for better exposition.

${ }^{2}$ Following the common convention, in the previous section we used $p$ to denote the rewiring probability in a small world network. From now on $p$ will denote price.
} 
The agents are myopic maximizers of the mean-variance expected utility:

$$
\max _{z_{t}}\left\{\mathrm{E}_{t-1}\left[W_{t+1}\right]-\frac{a}{2} \mathrm{~V}_{t-1}\left[W_{t+1}\right]\right\}
$$

where $a$ is the absolute risk aversion coefficient, and $\mathrm{E}_{t}$ and $\mathrm{V}_{t}$ denote conditional expectation and conditional variance that are based on the publicly available information set $I_{t}=\left\{p_{t}, p_{t-1}, p_{t-2}, \ldots ; y_{t}, y_{t-1}, y_{t-2}, \ldots\right\}$. The demand for the risky asset of the type $h$ agent is then given by:

$$
z_{t}^{h}\left(p_{t}\right)=\frac{\mathrm{E}_{t-1}^{h}\left[p_{t+1}+y_{t+1}\right]-R_{f} p_{t}}{a \mathrm{~V}_{t-1}^{h}\left[p_{t+1}+y_{t+1}\right]}=\frac{\mathrm{E}_{t-1}^{h}\left[p_{t+1}+y_{t+1}\right]-R_{f} p_{t}}{a \sigma^{2}} .
$$

Variables $\mathrm{E}_{t}^{h}$ and $\mathrm{V}_{t}^{h}$ are the expectations (or predictors) of the type $h$ agent about the mean and the variance, respectively. Note that we assume that all the agent types expect the same variance, $\mathrm{V}_{t}^{h}=\sigma^{2}$.

Suppose that the supply of outside shares of the risky asset $z^{s}$ is constant. Let $n_{t}^{h}$ be the fraction of type $h$ agents at date $t$, and $H$ be the number of trader types in the market. The equilibrium of supply and demand then results in the following pricing equation:

$$
\sum_{h=1}^{H} n_{t}^{h} \frac{\mathrm{E}_{t-1}^{h}\left[p_{t+1}+y_{t+1}\right]-R_{f} p_{t}}{a \sigma^{2}}=z^{s} .
$$

Under the assumption of zero total supply of the risky asset and homogeneous $(H=1)$ beliefs of the agents, the fundamental price $p^{*}$ is given by the discounted sum of the expected future dividends as a solution to the market-clearing equation (4), which is a well-known result. If, moreover, the dividend process is i.i.d. with constant mean $\bar{y}$, then $p^{*}=\bar{y} / r_{f}$.

We assume that there are two types of traders present in the market: fundamentalists and chartists. The fundamentalist traders forecast the next period price $p_{t+1}$ 
to equal the fundamental price $p^{*}$, that is:

$$
\mathrm{E}_{t-1}^{f}\left[p_{t+1}+y_{t+1}\right]=p^{*}+\bar{y}
$$

The chartists expect persistent deviations from the fundamental value of the price in the following form:

$$
\mathrm{E}_{t-1}^{c}\left[p_{t+1}+y_{t+1}\right]=p^{*}+g\left(p_{t-1}-p^{*}\right)+\bar{y}
$$

where $a>0$ is a constant and $g>0$ is an extrapolation parameter.

The belief types of agents are updated over time depending on the relative satisfaction from following a strategy of a specific type. The satisfaction has observed and unobserved components:

$$
\widetilde{U}_{t}^{h}=U_{t}^{h}+\frac{1}{\beta} \varepsilon_{t}^{h}
$$

where $U_{t}^{h}$ is the observed performance measure, $\varepsilon_{t}^{h}$ is the idiosyncratic noise (we later discuss its source), and $\beta$ is a coefficient, which controls the intensity of the noise. The observed performance measure $U_{t}^{h}$ is defined as a net profit of strategy $h$, that is

$$
U_{t}^{h}=\pi_{t}^{h}-C^{h}=\left(p_{t}+y_{t}-R_{f} p_{t-1}\right) z_{t-1}-C^{h},
$$

where $p_{t}+y_{t}-R_{f} p_{t-1}$ is the excess return earned per unit of the amount of the risky asset $z_{t-1}$ held in the agents' portfolio at the end of period $t-1$, and $C^{h}$ is the cost of following the strategy $h$. This cost is set to zero for the extrapolating rule of chartists and is strictly positive (set to unity) for fundamentalists.

In the $\mathrm{BH}$ model, where every agent knows the performance of both strategies, the dynamics of the model can be described through the co-evolution of the trader 


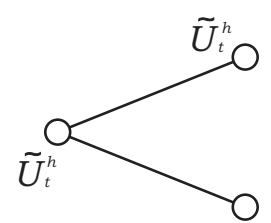

(a) Same type

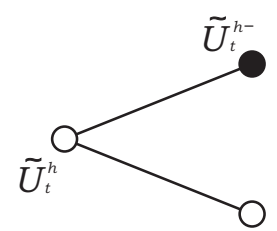

(b) Different type

Figure 3: Different neighbor types.

type fractions $n_{t}^{h}$ and the market equilibrium price. For a sufficiently large number of the agents, this fraction can be determined by $n_{t}^{h}=P\left(\widetilde{U}_{t}^{h}>\widetilde{U}_{t}^{h^{-}}\right)$, where $P$ denotes a probability measure. Moreover, if the idiosyncratic noise $\varepsilon_{t}^{h}$ in (7) follows the standard Gumbel (extreme value) distribution, by using the discrete choice model of Manski and McFadden (1990), $n_{t}^{h}$ can be directly expressed as

$$
n_{t}^{h}=\frac{\exp \left(\beta U_{t}^{h}\right)}{\exp \left(\beta U_{t}^{h}\right)+\exp \left(\beta U_{t}^{h^{-}}\right)} .
$$

In our setup the agents are located on the nodes of a network and can observe the performance measure of the strategies employed only by those agents who reside on the nodes directly connected with them. Hence, they cannot observe the strategy performance of the traders located two or more edges away. Therefore, contrary to Brock and Hommes (1998), we do not assume that the performance of every strategy is available to all the agents and we do not use the discrete choice fractions specified in (9). Instead, we allow only for local information exchange in the market. In particular, if an agent is surrounded by the agents of the same type (see Figure 3a), she does not switch as there is no information about the performance of the alternative strategy. If an agent has at least one neighbor of the different type ${ }^{3}$ (see Figure $3 \mathrm{~b}$ ), she is able to compare the satisfaction from her own strategy $\widetilde{U}_{t}^{h}$ with the satisfaction from the alternative strategy $\widetilde{U}_{t}^{h^{-}}$. She switches to the alternative

\footnotetext{
${ }^{3}$ If there are more than one neighbors of the different type, only one of them is consulted to compare strategies.
} 
strategy if it performs better. Formally, an agent chooses a strategy $h^{*}$ equal to

$$
h^{*}=\arg \max _{h, h^{-}}\left(\widetilde{U}_{t}^{h}, \widetilde{U}_{t}^{h^{-}}\right) .
$$

We allow for idiosyncratic noise across the agents in the performance measure, as specified by (7). The impact of the noise is inversely related to the intensity of the choice parameter $\beta$.

The noise in the performance measure may come from various sources. First, investors' decisions may be influenced by some unobserved factors in addition to the performance measure. These factors may be attributed to the cultural and historical characteristics (see Bond and Smith, 1996 and Hirshleifer, 2001) and differ across investors and across time. Second, the noise may be attributed to communication. We can distinguish two types of noise within this category. One type is attributed to plain noisy information transmission, i.e. under- or over-reporting of profits by neighbors due to behavioral biases. Another type of noise can be attributed to the amount of trust the agents put into the results communicated by their neighbors.

We assume that the noise term $\varepsilon_{t}^{h}$ in (7) is distributed according to the standard Gumbel distribution. Under this assumption, our model with a fully connected graph and a sufficiently large number of agents is comparable to the Brock and Hommes (1998) model.

Our market constitutes a complex adaptive dynamical system with co-evolving heterogeneous agents and equilibrium price. The model progresses in the following way (see Figure 4). After the expectations of the agents are formed and their demand is ascertained, the price is determined through the Walrasian auctioneer scenario. Next, the profits of the agents are determined. Then, agents compare their net profits with their neighbors' and switch to another strategy or remain with their own depending on their spatial position and relative performance. Finally the 
agents form their expectations again and the cycle repeats.

As we have discussed in Section 3, many

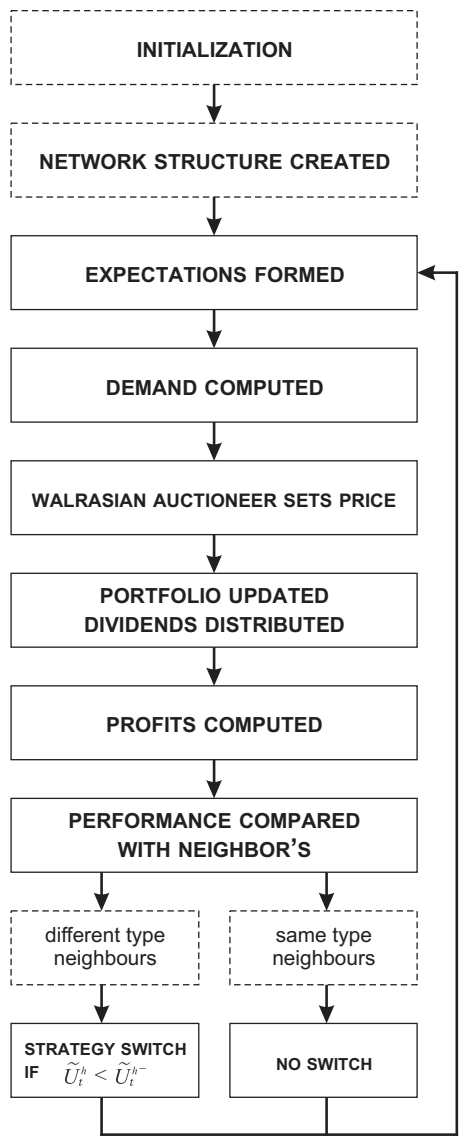

Figure 4: Temporal flow. studies point out that small world networks are good models for approximating real-life local interactions. In the next section we investigate the implications of introducing small world information exchange into the Brock and Hommes (1998) model. We also compare the effect from the small world network with other basic network topologies.

\section{Simulations and results}

By introducing nontrivial communication structures into the Brock and Hommes (1998) model we lose analytic tractability. Nevertheless, complex behavior of the resulting models can still be analyzed by means of computer simulations. ${ }^{4}$

We conduct simulations for four different network structures of local interactions, i.e. for a fully connected graph, a regular lattice, a small world graph with the rewiring probability equal to 0.01, and a random graph (see Figure 1). All the graphs are connected, that is, there are no nodes that do not have any edges. The fully connected graph is used as a benchmark corresponding to the finite number of agents implementation of the original Brock

\footnotetext{
${ }^{4}$ The $\mathrm{C}++$ code for our simulations is partially adapted from the code of Bottazzi et al. (2005).
} 


\begin{tabular}{lcc}
\hline Parameter & Symbol & Value/Range \\
\hline Intensity of choice & $\beta$ & {$[0.5,5]$} \\
Interest rate & $r_{f}$ & 0.1 \\
Mean dividend & $\bar{y}$ & 10 \\
Interpolation coefficient & $g$ & 1.4 \\
Initial price & $p_{0}$ & 103 \\
Initial fraction of fundamentalists & $n_{0}^{f}$ & 0.5 \\
Number of agents & $N$ & 1000 \\
Transient period & $T r$ & 2000 \\
Simulation length & $T$ & 2000 \\
\hline
\end{tabular}

Table 1: Parameter values used in simulations.

and Hommes (1998) model. We analyze the asset price dynamics for $N=1000$ agents. We found that for this number of agents fractions $n_{t}^{f}$ are close to the corresponding discrete choice probabilities in $(9) .{ }^{5}$ For comparison we choose the basic parameter values of the model similar to those used in Brock and Hommes (1998). The parameter values are summarized in Table 1.

\subsection{Evolution of prices and beliefs}

The asset price dynamics for a range of values of $\beta$ are shown by means of bifurcation diagrams in Figure 5. These bifurcation diagrams depict the dependence of the price distribution on the intensity of choice parameter $\beta$. The price distribution for each level of $\beta$ is represented by a gray-shade histogram. Darker shades correspond to areas of higher density. The histograms are computed using price levels from 10000 periods after 2000 transient periods with $\beta$ ranging from 0.5 to 5 and a linear step of 0.05

The two bifurcations occurring in the fully connected network are similar to the pitchfork and Neimark-Sacker bifurcations occurring in the Brock and Hommes (1998) model for $\beta^{*} \approx 2.3$ and $\beta^{* *} \approx 3.3$ respectively. During the pitchfork bifur-

\footnotetext{
${ }^{5}$ We also analyzed networks with $N=100$. Qualitatively the results were similar. However, the level of noise due to the finite sample implementation was much higher.
} 


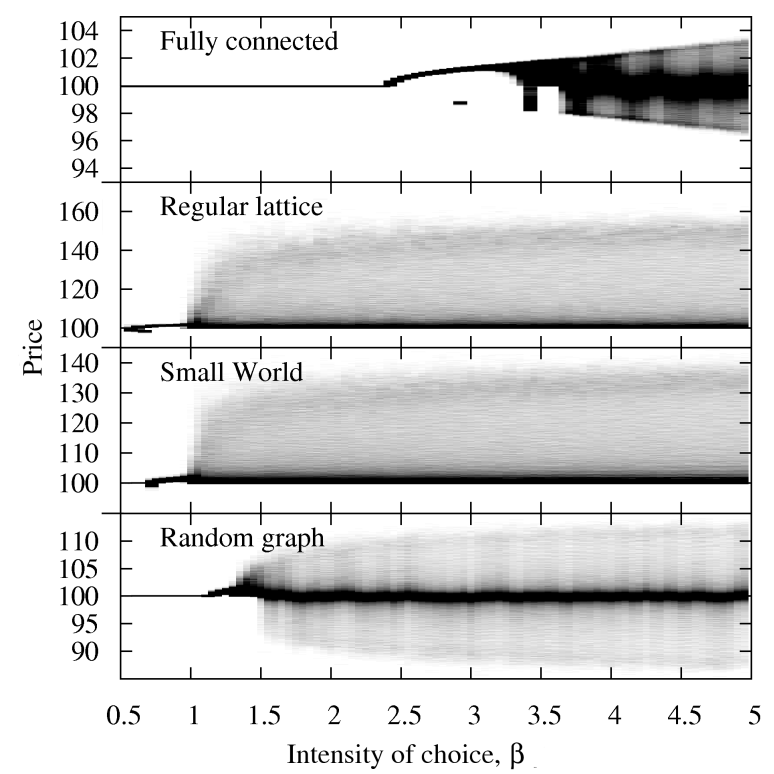

Figure 5: Bifurcation diagrams.

cation, the steady state loses its stability and two additional stable steady states are created. The Neimark-Sacker bifurcation leads to the emergence of periodic or quasi-periodic cycles. Economic intuition behind these bifurcations is as follows. The fundamentalists bring the price to the fundamental level, while the chartists destabilize the fundamental price by extrapolating the trend. The difference in the fractions of these two types determines the price behavior. When the price is close to the fundamental level the excess returns of the fundamentalists and the chartists are equal, but the former incur the costs. When $\beta<\beta^{*}$ this relative difference in past performance in not important for the choice of the forecasting rule. Thus, the difference in the fractions is not large enough and the price remains at the fundamental level. However, when $\beta^{*}<\beta<\beta^{* *}$, the relative past performance becomes more important and a larger fraction of agents chooses the less costly chartist rule. This results in the deviation of the equilibrium price from the fundamental level. When $\beta>\beta^{* *}$, that is, when the agents become highly reactive to the difference in excess returns, we observe cyclical behavior. When the price is near the fundamental 
level, the fraction of chartists rapidly increases amplifying any small deviations from the fundamental level and creating a bubble. The bubble ends since the extrapolative behaviour of chartists is not strong enough to sustain the trend and at some point fundamentalists start dominating the market bringing the price back to the fundamental level and the story repeats itself.

The values of $\beta$ for which two bifurcations occur in the fully connected network are close to the $\mathrm{BH}$ model. The other networks show somewhat different dynamics. The period between the primary and secondary bifurcations is shorter. In terms of the occurrence of the primary bifurcation with respect to the parameter $\beta$, the networks can be arranged in the following order (in decreasing value of $\beta$ ): the fully connected network (the benchmark), the random graph, the small world network, and the regular lattice. The same order holds with respect to the increase in the price amplitude. These results can be explained by the average speed of the information transmission between the agents within the network, which is closely related to the characteristic path length measure as discussed in Section 2. The information about the performance of the alternative strategy reaches all the nodes in the fully connected network within one time period. As we remove some edges, the information transmission between the agents who are not directly connected slows down. The speed of the information transmission is the slowest for the regular lattice. Slower information transmission results in higher persistence of one particular strategy over time, or, in other words, it delays the switching. Thus, the fraction of chartists becomes relatively large for smaller values of $\beta$ than in the fully connected network. This translates into earlier bifurcations. The post-bifurcation region of price instability becomes larger and the amplitude of price fluctuations becomes higher.

Figure 6 depicts the time series of the price for two values of the intensity of the switching parameter, $\beta=1$ and $\beta=3.5$, and the four networks: the fully 


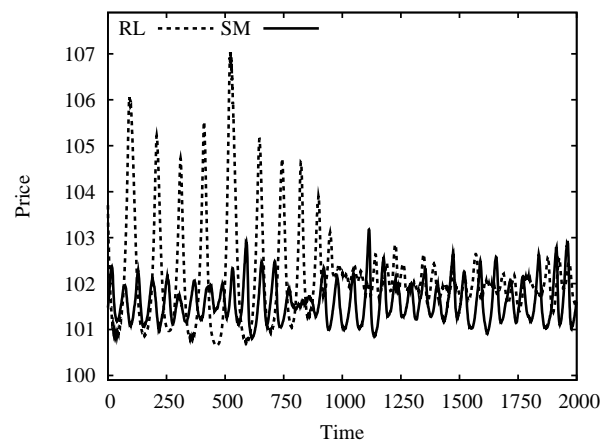

(a) $\beta=1$

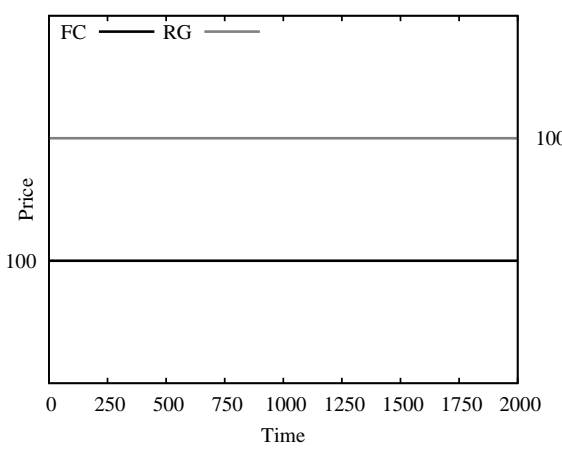

(c) $\beta=1$

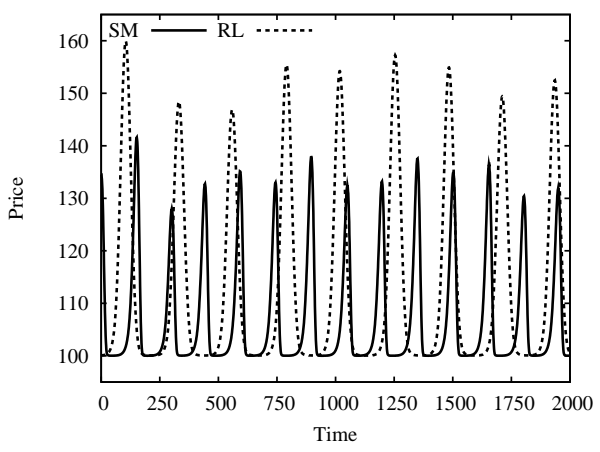

(b) $\beta=3.5$

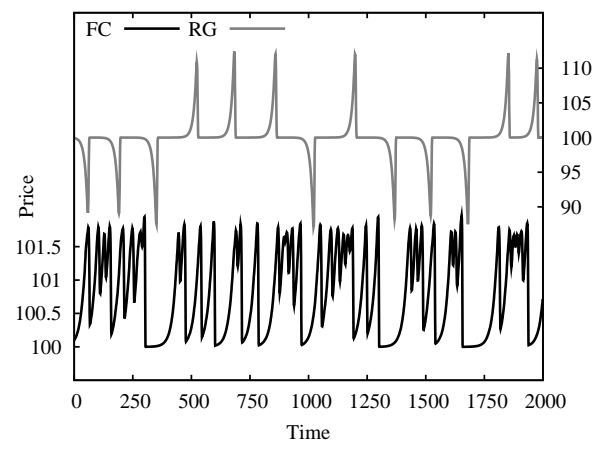

(d) $\beta=3.5$

Figure 6: Time series of price.

connected graph (FC), the regular lattice (RL), the small world network (SW) and the random graph (RG). We use this abbreviation in subsequent figures. For $\beta=1$ the price dynamics corresponding to the fully connected graph and the random network converges to a steady state, while the regular lattice and the small world network lead to highly irregular chaotic asset price fluctuations. For $\beta=3.5$, chaotic behavior is observed for all the network topologies, however, the regularity and the amplitudes of fluctuations vary considerably among them. The price dynamics of the random network are close to those of the fully connected network. The price dynamics produced by the regular lattice are the most distinct from the fully connected network. The small world network produces price dynamics similar to the regular lattice with some shift towards the random graph. 


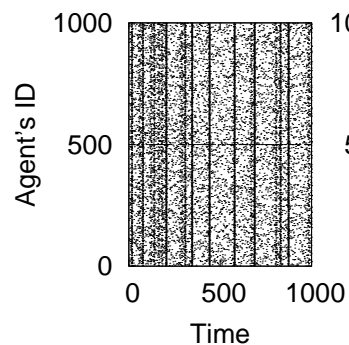

(a) Fully connected

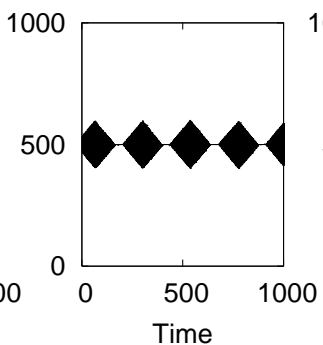

(b) Regular lattice

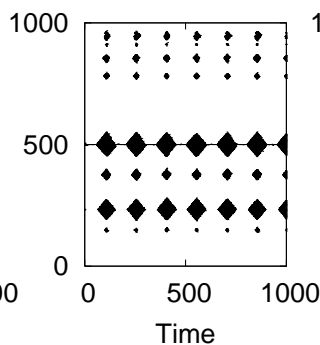

(c) Small world

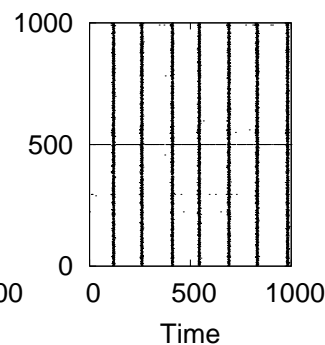

(d) Random graph

Figure 7: Evolution of agent population.

To provide insights into the effects of different network topologies on market behavior we track how individual agents change their forecasting beliefs over time. Figure 7 shows a typical set of patterns that emerge during simulations. This set is for $\beta=3.5$. The figure shows the evolution of the forecasting type for all 1000 agents at every time step from 0 to 1000. Each point on a vertical line represents an agents' type: a black point indicates the fundamentalist type, while a blank (or white) point indicates the chartist type. As reference points about the performance of the alternative strategy, two agents in our system do not change their types: agent 500 is always a fundamentalist, while agent 0 is a chartist. This is done to prevent the possible extinction of one of the agents' types, which may happen in our model because of the finite number of agents. ${ }^{6}$ The periods of the highest concentration of fundamentalists correspond to the time when the price falls to the fundamental level, while the lowest concentration of fundamentalists corresponds to the highest deviation from the fundamental value of the price. Overall, the fraction of fundamentalists is higher in the fully connected network. This is consistent with smaller deviations from the fundamental price and frequent price oscillations. Fundamentalist are uniformly distributed across the network. In the case of regular lattice we observe high clustering of the fundamentalists around the fundamental "core".

\footnotetext{
${ }^{6}$ In the Brock and Hommes (1998) model the fractions of the both types are always positive, but may be very small. Since their model assumes infinitely large number of agents, the extinction does not occur.
} 


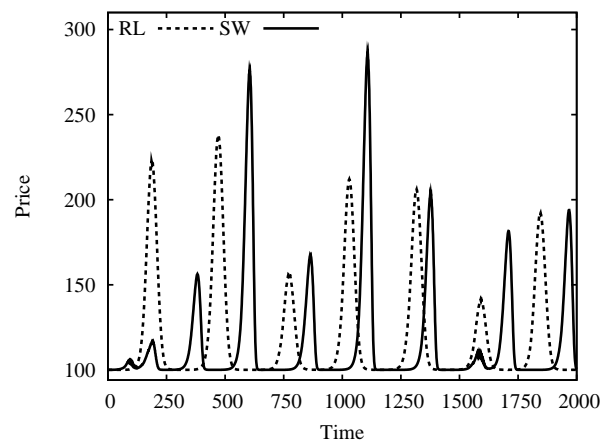

(a) $\beta=1$

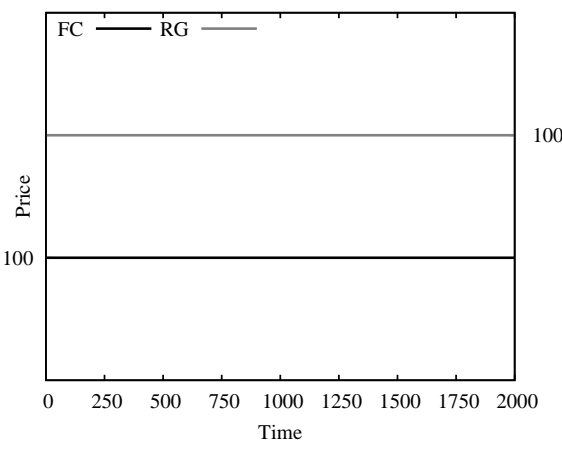

(c) $\beta=1$

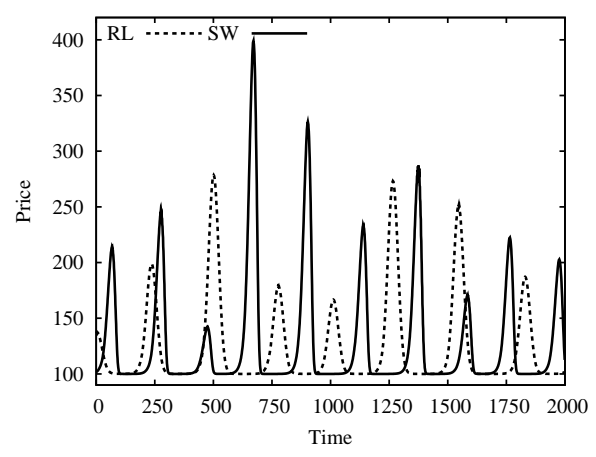

(b) $\beta=3.5$

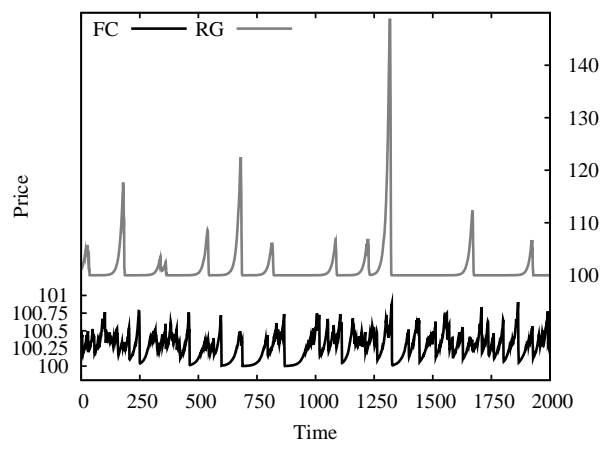

(d) $\beta=3.5$

Figure 8: Time series of price with a stochastic dividend process.

This is consistent with the high clustering coefficient of this network. In the small world network we also observe clusters, but they are smaller and more disperse in space. Again this is consistent with sparsity and a high clustering coefficient typical for this network. In the case of random graph we do not observe any clusters of the fundamentalists. This is due to a very small clustering coefficient for this network and a relatively small number of the fundamentalists on the market during most of the periods.

Figure 8 presents time series plots for the asset price corresponding to the case of stochastic dividends. We assume that the dividends are independent and identically normally distributed with the mean set to 10 and the variance of 1 . For $\beta=1$, even with the small variance in the dividend process, the time series of the small world 


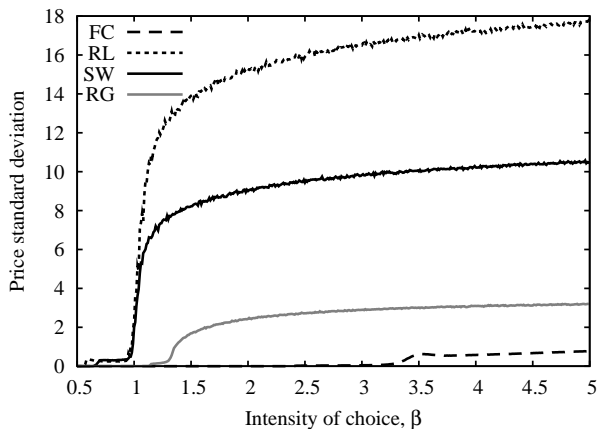

(a) Standard deviation of price

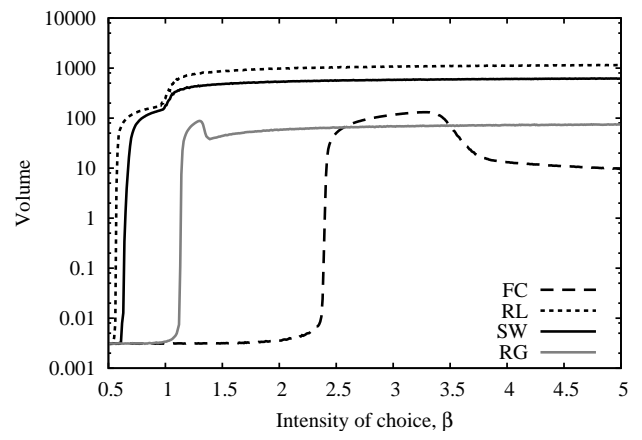

(b) Average traded volume

Figure 9: Measures of market information inefficiency.

network and the regular lattice exhibit large fluctuation. For the fully connected network and the random graph the price stays at the fundamental level of 100. For $\beta=3.5$, the regular lattice and the small world network produce even greater irregular single-peak deviations from the fundamental price, while the fully connected and the random networks produce relatively regular fluctuations of a much smaller scale. A comparison of Figure 6 with Figure 8 suggests that the impact of the stochastic dividend on the price is the strongest in the small world and the regular lattice networks.

The informational efficiency is closely related to the speed of information transmission (or characteristic path length) and can be measured by comparing the volatility of the observed price with the volatility of the fundamental dividend process as suggested by Shiller (1981). In order to abstract from the effect of timevarying dividend in our model, we keep the dividend process constant. Under this assumption, the Efficient Market Hypothesis would predict constant price over time and zero trading volume. In Figure 9 we analyze the standard deviation of the price (panel a) and the average traded volume (panel b) for values of $\beta$ ranging from 0.5 to 5 for the four topologies. We ignore the first 2000 transitory iterations and compute the standard deviation of price and the average traded volume for the following 2000 


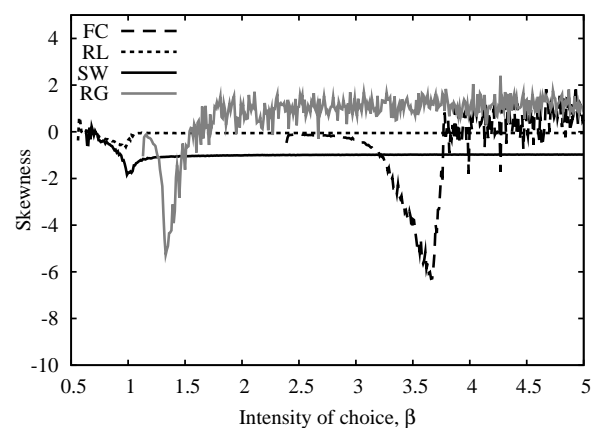

(a) Distribution skewness

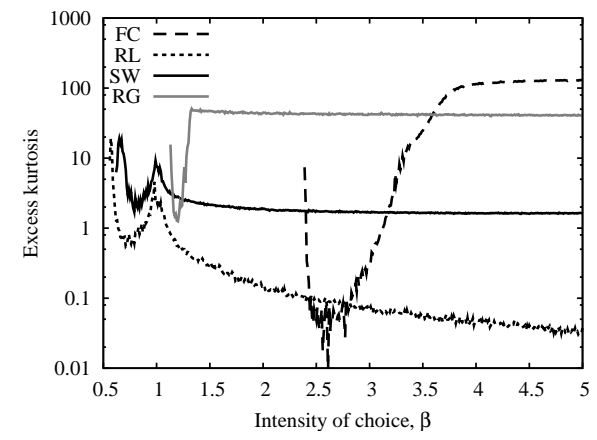

(b) Excess kurtosis

Figure 10: Skewness and kurtosis of returns.

periods. To eliminate the dependence of our results on a particular realization of the random seed, we report averages for 100 simulation runs, each run having its own random seed. The same simulation setup is used for all the other statistics reported further. We observe that the random graph and the fully connected network exhibit the most informational efficient outcomes for any values of $\beta$ which is consistent with the highest speed of information transmission in these two networks. The regular lattice exhibits the least informationally efficient outcome.

\subsection{Statistical properties}

Below we analyze the properties of the time series generated by the four considered networks. This analysis helps us to understand which network structure generates the time series, properties of which are closer to the stylized facts observed in reality.

Figure 10 depicts the skewness of the returns and the excess kurtosis of the returns. The former statistic (Figure 10a) measures the asymmetry of the distribution. It is close to zero for all the networks for all post-bifurcation values of $\beta$. The returns generated by the model with the small world network are slightly negatively skewed.

The excess kurtosis plot (Figure 10b) reveals that all the four networks generate 


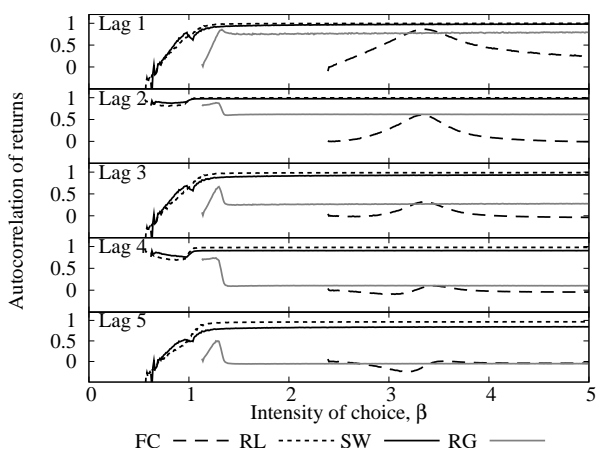

(a) Autocorrelations of returns

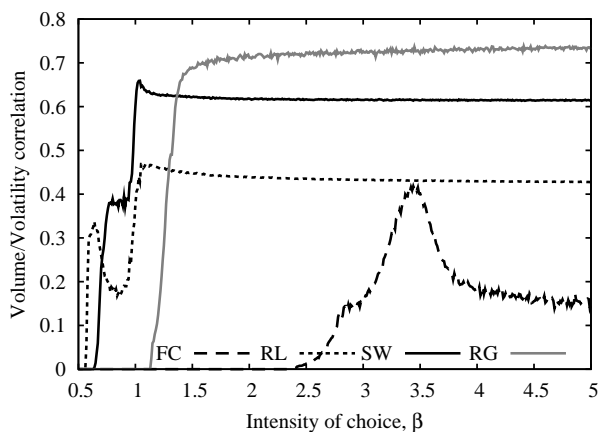

(b) Volume/volatility correlations

Figure 11: Properties of returns and volume.

return distributions with different kurtosis values. The small world network return distribution exhibits the excess kurtosis value close to 5 , which is close to the one observed for the returns on the real financial markets.

By computing the autocorrelation of the returns for the four network structures, we can analyze linear unpredictability of the stock returns, which is a well-known property of the time series exhibited by the real financial markets. Figure 11a depicts the autocorrelation of returns for the first five lags as a function of the intensity of choice. Usually the real financial time series exhibit small or no autocorrelation of returns. The regular lattice and the small world network produce high autocorrelations at all lags. This, again, can be attributed to a less efficient information transmission in these networks. Although the random graph and the fully connected network display large autocorrelations at the first two lags, they converge to zero autocorrelation values at lag three to five. The significant positive autocorrelations are resulting predominantly from the persistence of chartists strategies. It is possible to reduce the autocorrelations by adding a sufficient amount of dynamical noise into the price as in Hommes (2002). However, we do not aim to reproduce stylized facts in this paper and therefore do not pursue this route.

Figure $11 \mathrm{~b}$ shows the correlations between the squared returns and the volume 


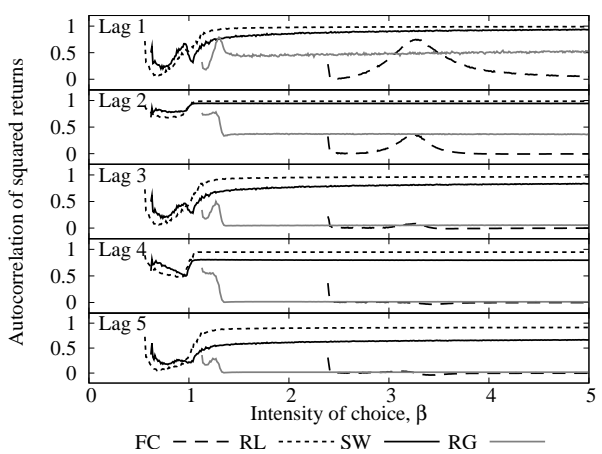

(a) Autocorrelations

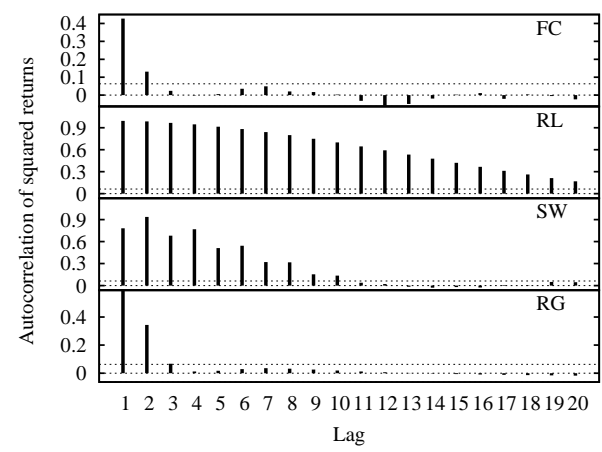

(b) $\mathrm{ACF}$

Figure 12: Autocorrelation of squared returns.

of trades. In real financial markets, high trade volumes are associated with high volatility. Many standard asset pricing models, however, fail to reproduce this fact. Our model produces positive volume-volatility correlations for all networks. The highest values in the post-bifurcation region are observed under the random graph network, followed by the small world network.

A universal property of the real financial time series is the volatility clustering, i.e., the presence of slow decaying autocorrelations in the squared returns. Figure 12a shows the autocorrelations of the squared returns at the first five lags as a function of $\beta$, while Figure $12 \mathrm{~b}$ shows the autocorrelation function of the squared returns for 20 lags with $\beta=3.5$. The autocorrelations of the squared returns under the fully connected network and random graph vanish after first few lags, which is not consistent with stylized facts. In turn the autocorrelations under the regular lattice and the small world network remain positive and large at many lags for the regular lattice and the small world network indicating the volatility clustering of the returns.

The above analysis reveals that different local interaction arrangements in the market affect the dynamics and the time series properties. The effect of the change in the behavior parameter $\beta$ also depends on a particular network configuration. 


\section{Concluding remarks}

In this paper we expanded the model of Brock and Hommes (1998) by introducing local information exchange via communication networks. We studied how different network structures affect asset price dynamics. We observed that the stability region with respect to the intensity of choice parameter $\beta$ depends on the communication network. A relatively slower information transmission in the regular lattice and the small world networks creates greater information inefficiencies and induces greater instabilities and higher deviations in the price dynamics. Upon the analysis of the statistical properties of the time series of the returns generated by different networks we observed that the asset price dynamics generated under the small world network exhibit some properties close to those observed in the real financial markets.

In many networks there is a feedback between network performance and network formation. This means that the performance of agents also influences the network topology they are active in. For simplicity in this work we imposed the network structure on the agents. Endogenous network formation is an extension which we plan to address in the future work.

\section{References}

Albert, Réka and Barabási, Albert-László, 2002. Statistical mechanics of complex networks. Reviews of Modern Physics, 74, 47-97.

Alfarano, Simone and Milaković, Mishael, 2009. Network structure and Ndependence in agent-based herding models. Journal of Economic Dynamics and Control, 33, 78-92.

Anufriev, Mikhail and Bottazzi, Giulio, 2004. Asset pricing model with heteroge- 
neous investment horizons. Laboratory of Economics and Management Working Paper Series, Sant'Anna School for Advanced Studies.

Anufriev, Mikhail and Panchenko, Valentyn, 2006. Heterogeneous Beliefs under Different Market Architectures. Advances in Artificial Economics: The Economy as a Complex Dynamic System, volume 584, pp. 3-15. Springer.

Arnswald, Torsten, 2001. Investment behaviour of German equity fund managers - an exploratory analysis of survey data. Deutsche Bundesbank Working Paper $08 / 01$.

Arthur, W. Brian, Holland, John H., LeBaron, Blake, Palmer, Richard and Taylor, Paul, 1997. Asset Pricing Under Endogenous Expectation in an Artificial Stock Market. The Economy as an Evolving Complex System, volume II, pp. 15-44. Perseus Books.

Baum, Joel A. C., Shipilov, Andrew V. and Rowley, Tim J., 2003. Where do small worlds come from? Industrial and Corporate Change, 12, 697-725.

Bond, Rod and Smith, Peter B., 1996. Culture and conformity: A meta-analysis of studies using Asch's (1952b, 1956) line judgment task. Psychological Bulletin, $119,111-137$.

Boswijk, H. Peter, Hommes, Cars H. and Manzan, Sebastiano, 2007. Behavioral heterogeneity in stock prices. Journal of Economic Dynamics and Control, 31, 1938-1970.

Bottazzi, Giulio, Dosi, Giovanni and Rebesco, Igor, 2005. Institutional architectures and behavioral ecologies in the dynamics of financial markets. Journal of Mathematical Economics, 41, 197-228. 
Brock, William, Hommes, Cars H. and Wagener, Florian, 2006. More hedging instruments may destablize markets. CeNDEF Working Paper Series, University of Amsterdam.

Brock, William A. and Durlauf, Steven N., 2001. Discrete choice with social interactions. Review of Economic Studies, 68, 235-260.

Brock, William A. and Hommes, Cars H., 1998. Heterogeneous beliefs and routes to chaos in a simple asset pricing model. Journal of Economic Dynamics and Control, 22, 1235-1274.

Brock, William A., Hommes, Cars H. and Wagener, Florian O. O., 2005. Evolutionary dynamics in markets with many trader types. Journal of Mathematical Economics, 41, 7-42.

Chang, Sheng-Kai, 2007. A simple asset pricing model with social interactions and heterogeneous beliefs. Journal of Economic Dynamics and Control, 31, 1300-1325.

Chen, Shu-Heng, Lux, Thomas and Marchesi, Michele, 2001. Testing for non-linear structure in an artificial financial market. Journal of Economic Behavior \& Organization, 46, 327-342.

Chen, Shu-Heng and Yeh, Chia-Hsuan, 2001. Evolving traders and the business school with genetic programming: A new architecture of the agent-based artificial stock market. Journal of Economic Dynamics and Control, 25, 281-654.

Chiarella, Carl, 1992. The dynamics of speculative behaviour. Annals of Operations Research, 37, 101-123.

Davis, Gerald, Yoo, Mina and Baker, Wayne, 2003. The small world of the American corporate elite, 1982-2001. Strategic Organization, 1, 301-326. 
Day, Richard and Huang, Weihong, 1990. Bulls, bears and market sheep. Journal of Economic Behavior \& Organization, 14, 299-329.

de Fontnouvelle, Patrick, 2000. Information dynamics in financial markets. Macroeconomic Dynamics, 4, 139-169.

DeLong, J. Bradford, Shleifer, Andrei, Summers, Lawrence H. and Waldmann, Robert J., 1990. Noise trader risk in financial markets. Journal of Political Economy, 98, 703-738.

Diks, Cees and van der Weide, Roy, 2005. Herding, a-synchronous updating and heterogeneity in memory in a CBS. Journal of Economic Dynamics and Control, $29,741-763$.

Duffy, John, 2001. Learning to speculate: Experiments with artificial and real agents. Journal of Economic Dynamics and Control, 25, 281-654.

Duflo, Esther and Saez, Emmanuel, 2002. Participation and investment decisions in a retirement plan: The influence of colleagues' choices. Journal of Public Economics, 85, 121-148.

Ehrentreich, Norman, 2006. Technical trading in the Santa Fe Institute artificial stock market revisited. Journal of Economic Behavior \& Organization, 61, 599616.

Frankel, Jeffrey A. and Froot, Kenneth A., 1987. Using survey data to test standard propositions regarding exchange rate expectations. American Economic Review, 77, 133-153.

Friedman, Milton, 1953. The Case for Flexible Exchange Rates. Essays in Positive Economics, pp. 157-203. University of Chicago Press. 
Gerasymchuk, Sergiy, 2008. Reference dependence and social interactions in agentbased models of financial markets. Ph.D. Thesis. Advanced School of Economics, University of Venice.

Gode, Dhananjay K. and Sunder, Shyam, 1997. What makes markets allocationally efficient? The Quarterly Journal of Economics, 112, 603-630.

Hirshleifer, David, 2001. Investor psychology and asset pricing. The Journal of Finance, 56, 1533-1597.

Hommes, Cars H., 2001. Financial markets as complex adaptive evolutionary systems. Quantitative Finance, 1, 149-167.

Hommes, Cars H., 2002. Modeling the stylized facts in finance through simple nonlinear adaptive systems. Proceedings of the National Academy of Sciences of the United States of America, 99, 7221-7228.

Hommes, Cars H., 2006. Heterogeneous Agent Models in Economics and Finance. Handbook of Computational Economics: Agent-Based Computational Economics, volume 2, pp. 1109-1186. Elsevier/North-Holland.

Hommes, Cars H., Huang, Hai and Wang, Duo, 2005. A robust rational route to randomness in a simple asset pricing model. Journal of Economic Dynamics and Control, 29, 1043-1072.

Hong, Harrison, Kubik, Jeffrey D. and Stein, Jeremy C., 2004. Social interaction and stock-market participation. The Journal of Finance, 59, 137-163.

Hong, Harrison, Kubik, Jeffrey D. and Stein, Jeremy C., 2005. Thy neighbor's portfolio: Word-of-mouth effects in the holdings and trades of money managers. The Journal of Finance, 60, 2801-2824. 
Ito, Takatoshi, 1990. Foreign exchange rate expectations: Micro survey data. American Economic Review, 80, 434-449.

Kirman, Alan, 1993. Ants, rationality, and recruitment. The Quarterly Journal of Economics, 108, 137-156.

LeBaron, Blake, 2006. Agent-based Computational Finance. Handbook of Computational Economics: Agent-Based Computational Economics, volume 2, pp. 1187-1234. Elsevier/North-Holland.

LeBaron, Blake, Arthur, W. Brian and Palmer, Richard, 1999. Time series properties of an artificial stock market. Journal of Economic Dynamics and Control, 23, $1487-1516$.

Lux, Thomas, 1995. Herd behaviour, bubbles and crashes. The Economic Journal, $105,881-896$.

Madrian, Bridget and Shea, Dennis, 2000. Peer effects and savings behavior in employer-sponsored savings plans. University of Chicago working paper.

Manski, Charles F. and McFadden, Daniel, 1990. Structural Analysis of Discrete Data with Econometric Applications, Fourth edn. MIT Press.

Morone, Piergiuseppe and Taylor, Richard, 2004. Knowledge diffusion dynamics and network properties of face-to-face interactions. Journal of Evolutionary Economics, 14, 327-351.

Shiller, Robert J., 1981. Do stock prices move too much to be justified by subsequent changes in dividends? American Economic Review, 71, 421-436.

Shiller, Robert J., 1995. Conversation, information, and herd behavior. American Economic Review, 85, 181-85. 
Shiller, Robert J. and Pound, John, 1989. Survey evidence on diffusion of interest and information among investors. Journal of Economic Behavior \& Organization, $12,47-66$.

Valente, Thomas W. and Davis, Rebecca L., 1999. Accelerating the diffusion of innovations using opinion leaders. The Annals of the American Academy of Political and Social Science, 566, 55-67.

Wasserman, Stanley and Faust, Katherine, 1994. Social Network Analysis: Methods and Applications. Cambridge University Press.

Watts, Duncan J., 1999. Small Worlds. Princeton University Press.

Watts, Duncan J. and Strogatz, Steven H., 1998. Collective dynamics of "smallworld" networks. Nature, 393, 440-442. 